\title{
Histone methylations in the developing central nervous system and in neural tube defects
}

https://doi.org/10.1515/nf-2018-A001

\begin{abstract}
Understanding central nervous system genesis is of crucial relevance to decode different human diseases such as microcephaly or neural tube defects, which arise from incorrect developmental processes. Epigenetic mechanisms regulate gene expression in a spatio-temporal manner and are implicated in diverse cellular actions one of which is cell differentiation. Therefore, the study of these mechanisms is of great relevance in the context of development and disease. In this article, we will review histone methylations as epigenetic modification and how they impact on gene expression and cell differentiation in central nervous system development and neural differentiation. Further, we will discuss an emerging link between histone methylation in the etiology of neural tube defects. We will specifically highlight the role of the disruptor of telomeric silencing like 1 (DOT1L) and histone H3 lysine 79 methylation (H3K79me), which is an unusual histone modification with implication for proper central nervous system development.
\end{abstract}

Keywords: Development; Neural Differentiation; Epigenetics; Histone Methylation; Neural Tube Defects

\section{Introduction}

In a multicellular organism like human beings, all cells in the body originate from one single cell, the zygote, which results from the fusion between egg and sperm. After fertilization, the zygote will proliferate giving rise to many other cells, tissues, and organs until reaching a complete body in a process named embryogenesis. One of the hallmarks during embryogenesis is the gastrulation step, in which the three germ layers are formed (Kiecker et al.,

*Corresponding author: Tanja Vogel, Institute for Anatomy and Cell Biology, Department of Molecular Embryology, Medical Faculty, Albert-Ludwigs-University Freiburg, 79104 Freiburg, Germany, Mail: Tanja.Vogel@anat.uni-freiburg.de

Alejandro Villarreal, Henriette Franz, Institute for Anatomy and Cell Biology, Department of Molecular Embryology, Medical Faculty, Albert-Ludwigs-University Freiburg, 79104 Freiburg, Germany, Mail: Alejandro.Villarreal@anat.uni-freiburg.de, Henriette.Franz@anat. uni-freiburg.de
2016; Solnica-Krezel and Sepich, 2012; Tseng et al., 2017). These layers are endoderm, mesoderm and ectoderm. Further on during development, the ectoderm will give rise to the neural tube, from where the central nervous system derives (Greene and Copp, 2014; Smith and Schoenwolf, 1997) (Figure 1A). The central nervous system comprises two gross anatomical structures: the brain and the spinal cord. These structures contain multiple types of neurons, which transmit and receive electric signals throughout the body. Besides the neurons, astrocytes, oligodendrocytes and microglial cells are also part of the central nervous system (Figure 1B). Astrocytes provide metabolic support to neurons, regulate synaptic transmission, are involved in the formation of the blood brain barrier and have neuroprotective effects during brain injury. Oligodendrocytes generate the myelin sheets around neuronal axons. An interesting fact is that, except for microglial cells, which derive from precursors originating in the yolk sac, all the other cell types share a common origin in the central nervous system. That means that they derive from a common precursor cell. These initially multipotent precursors are the neural stem cells that reside in the neural tube. During central nervous system development, the neural stem cells adapt the potential to either proliferate to produce more stem cells or to enter differentiation in the consecutive processes of neuro-, astro- or oligodendrogenesis. Whereas proliferative cell division leads to a daughter cell generation with the same developmental potential, differentiative cell division comes along with a restricted potential and the daughter cell is determined to adapt a certain cell fate. Specific signals are needed to stop the proliferation and to start differentiation. Thus, the neural stem cell gradually restricts and redirects its proliferative or differentiative potential, a phenomenon called cell commitment (Hirabayashi and Gotoh, 2010; Qiao et al., 2016; Tang et al., 2015).

But what makes a neuron to be a neuron, an astrocyte to be an astrocyte and an oligodendrocyte to be what it needs to be? How does a cell know which phenotype and function to adopt? How is a fate maintained and not reverted? Since years, we know that the activity or transcription of specific genes determine cell fate and function. All cells originated from the zygote, and therefore all cells have the same DNA sequence and composition of 
A

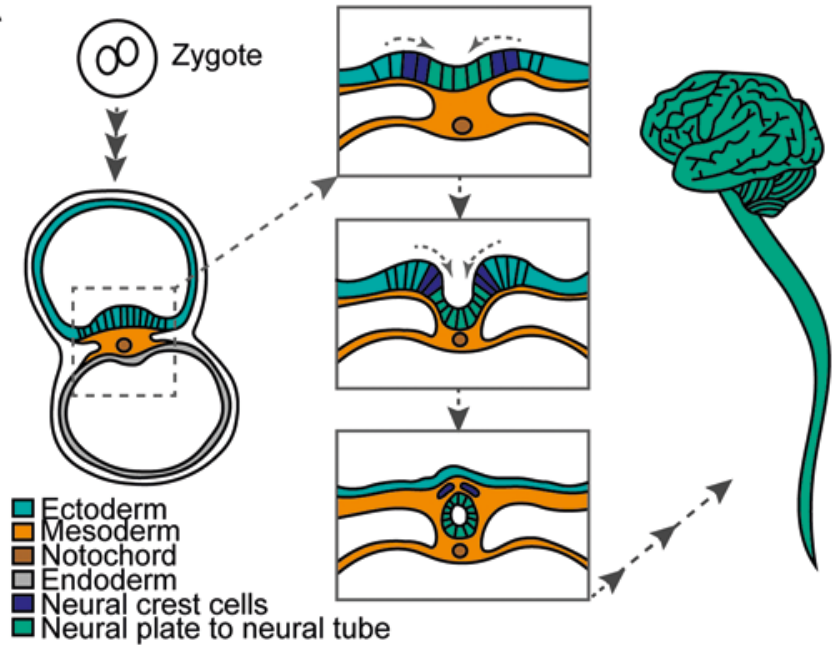

B

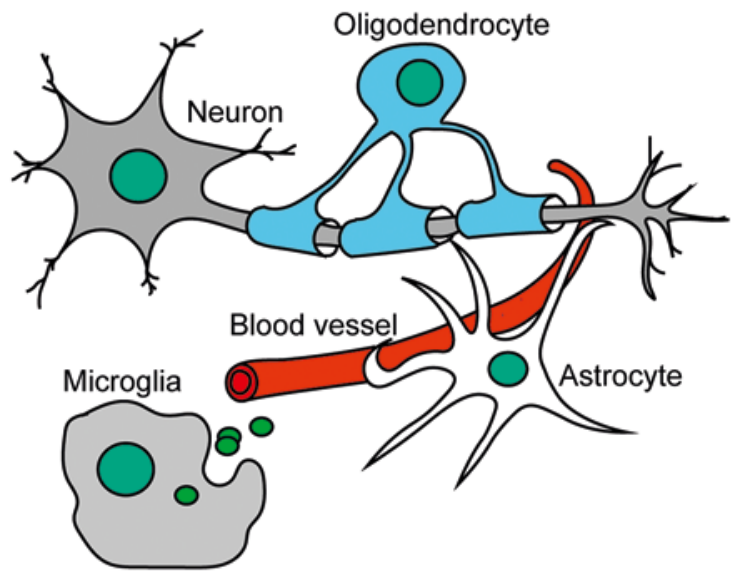

Fig. 1: A. Development from zygote over neural tube to CNS in humans. B. Neurons, Astrocytes, oligodendrocytes and microglia are main cell types of the CNS.

genes. But to drive different cell fates it is necessary that specific subsets of genes are active at precise time points. Thus, it is important to understand how the subsets of genes are selected and how the expression of these genes is activated, repressed or balanced to adapt a different cell fate. There is a plethora of molecular mechanisms known to be involved in the regulation of gene transcription. In this review, we will focus solely on epigenetic mechanisms that include histone modifications and how these protein modifications influence gene transcription in the context of cell differentiation in the central nervous system.

\section{Structure of the chromatin}

Histones are small proteins that have the capacity to bind DNA. The structural unit of histone proteins and DNA is called chromatin. Two of each core histones, H2A, H2B, $\mathrm{H} 3$ and $\mathrm{H} 4$ associate to form an octamer. $146 \mathrm{bp}$ of the DNA wraps approx. 1,5 times around each histone octamer. This smallest compaction unit of the chromatin, the DNA-histone core complex, is called nucleosome. A fifth type of histone localises outside of the nucleosome. This is the linker histone $\mathrm{H} 1$ that serves on a higher level to compact the DNA (Cutter and Hayes, 2015; Lawrence et al., 2016). Histones undergo chemical modifications after they are synthesized, as other proteins in the cell. The process of protein synthesis is called translation and the subsequent chemical changes of proteins are known as posttranslational modifications. Posttranslational modifications induce different states of a protein that allow e.g. for dif- ferent enzymatic activity, protein interactions, or localisation within a specific cellular compartment. Among the posttranslational modifications of histones the following have been widely studied: acetylation, phosphorylation, methylations, deamination, ubiquitination, ADP ribosylation and sumoylation (Lawrence et al., 2016; Peserico and Simone, 2011; Prachayasittikul et al., 2017; Yang and Seto, 2007). Posttranslational modifications of histones are epigenetic modifications as they have the potential to be transmitted into the next cell generation and they carry specific information about the transcriptional status of the associated genomic locus. They are set by enzymes, the writers, interpreted by binding proteins, the readers, and removed by erasers (Figure 2A). Therefore, posttranslational modifications can be highly flexible and provide another layer of genetic information extending the genetic code. The different posttranslational modifications on the histones affect e.g. the structure of the chromatin by either making it more (open chromatin) or less (closed chromatin) accessible for the transcriptional machinery. This mechanism is very important since open chromatin usually contains genes that are actively transcribed. On the other hand, if the chromatin has a closed configuration, genes will be silent and not transcribed. Open and active chromatin is known as euchromatin, whereas silent chromatin is called heterochromatin (Cutter and Hayes, 2015; Luger et al., 2012; Venkatesh and Workman, 2015). 

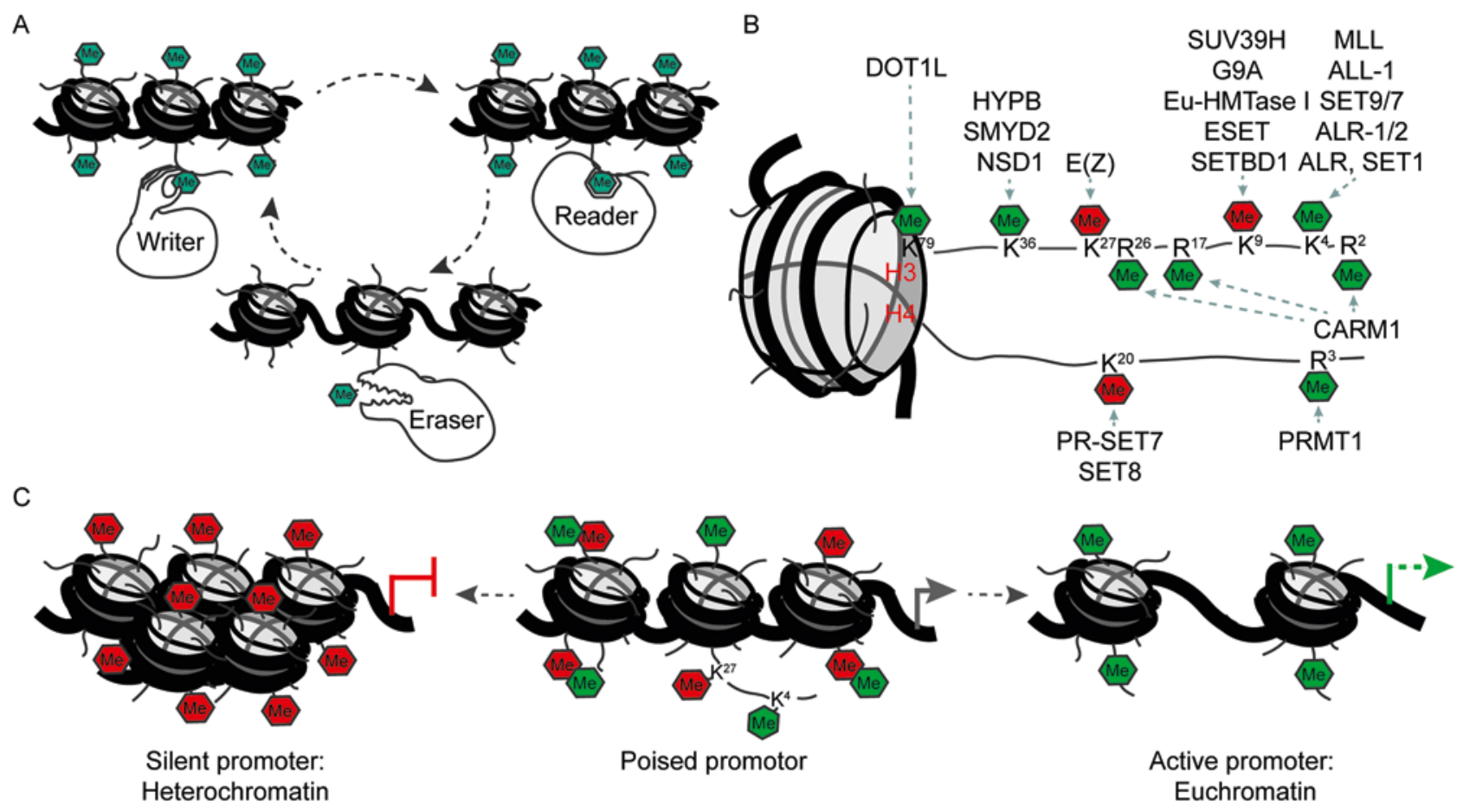

Fig. 2: A. Writer, reader and eraser proteins of histone modifications. B. Histone methylation marks and their writing enzymes. Green: activation of transcription. Red: repression of transcription. C. Poised promoters carry activating and repressing modifications at the same time. During development of specific cell types one of the modifications remains and the respective gene will be activated or repressed.

\section{Regulation of chromatin accessibi- lity by histone modifications}

Histones can be modified at specific amino acids. Both the position of the modified amino acids in the histone as well as the chemical group that is attached to the amino acid bear information on chromatin accessibility. Posttranslational modifications can alter the interaction between the positively charged histones and the negatively charged phosphate groups of the DNA. Chromatin will become open and accessible to transcription factors if the interaction is less strong, e.g. through addition of negative charges into the histone. This is achieved if a histone becomes for example acetylated. Acetylation of a histone will thus reduce stability of the whole nucleosome. Therefore, histone acetylation is transcriptionally activating.

The outcome of histone methylations with regard to transcriptional activity is more diverse. Depending on the position of the amino acid, a methylation can result in the activation or repression of a gene (Figure 2B). Lysine (abbreviated as K) can be mono, di and trimethylated (me1, me2 and me3), while arginine (R) only carries me1 and me2 for steric reasons. Not only the degree of methylation but also the position of the amino acid influences the outcome for gene activity. For example, trimethylation of histone 3 at lysine 9 or lysine 27 (H3K9me3 and H3K27me3, respectively), results in repression of gene expression. On the other hand, H3K4me3 and H3K36me3 participate in transcriptional initiation and elongation (Lawrence et al., 2016; Peterson and Laniel, 2004). A further variability is the position of the histone methylation within a gene. For example, H3K4me1 is located at genomic regions which increase gene transcription, known as enhancers, whereas $\mathrm{H} 3 \mathrm{~K} 4 \mathrm{me} 3$ is present at genomic regions which initiate the transcription of a gene, known as active promoters (Heintzman et al., 2007; Shlyueva et al., 2014). The combination of all histone modifications within a genome establishes a "histone code" which determines the transcription of specific genes and thereby cellular commitment.

Especially during development, active and repressive histone modifications sometimes coexist at the same regulative elements. For example, if such a histone code is located in the promoter region upstream of the transcriptional start site, this promoter is called "poised". The reason for this is that these promoters, which are marked at the same time with the activating H3K4me3 and the repressing H3K27me3 or H3K9me3, respectively, are within a so called bivalent state (Figure $2 \mathrm{C}$ ). The downstream gene of poised promoters will generally be not active. However, 
A

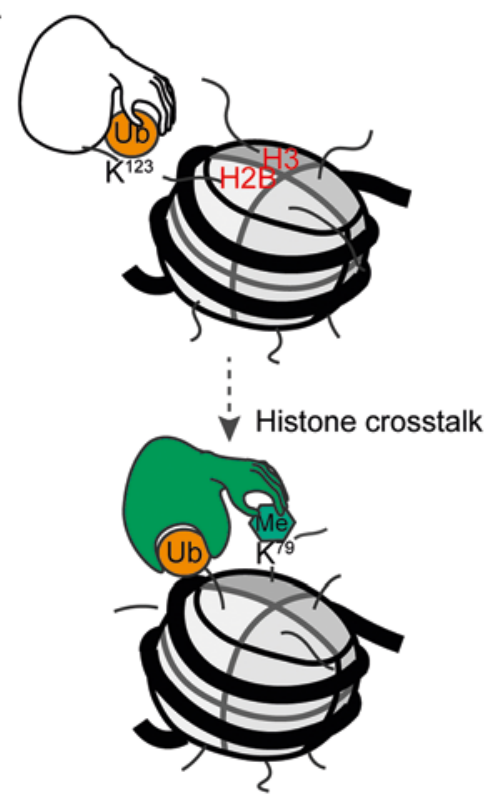

C

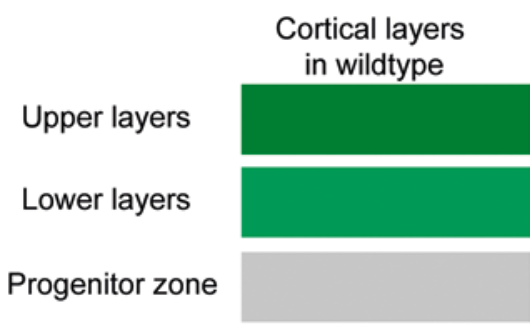

B
Neurogenin 1 transcriptionally active in neural stem cells

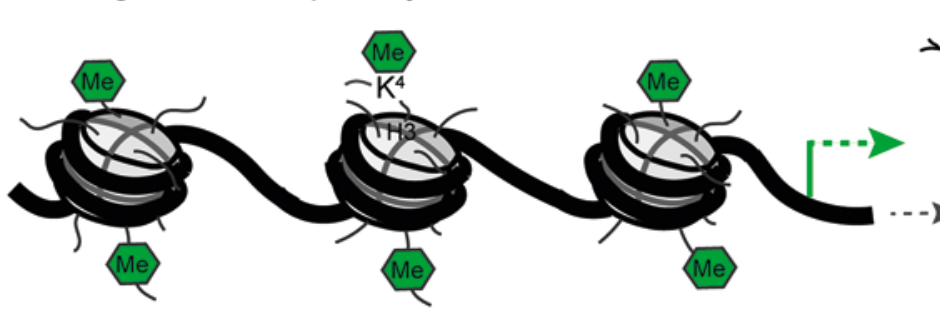

Neurogenin1 transcriptionally silent in neural stem cells
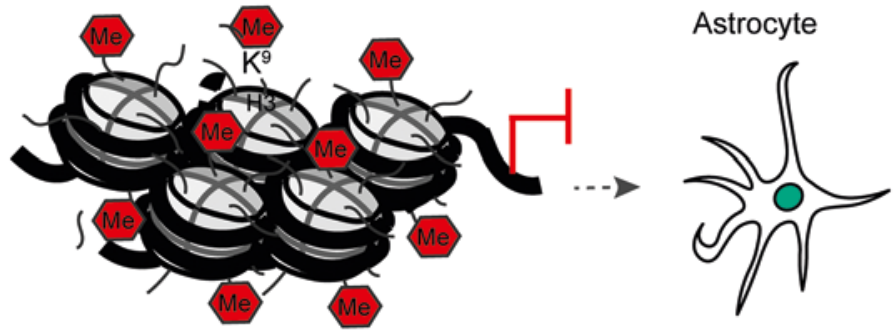

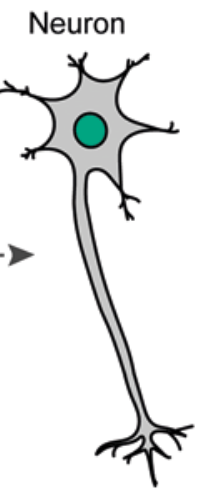

Cortical layers in DOT1L-CKO/AF9-cKO

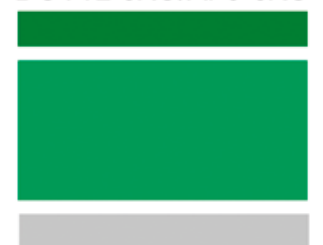

Fig. 3: A. Example of histone crosstalk: if H2B lysine 123 is ubiquitinated (H2Bub), methylation of H3 lysine 79 (H3K79) can follow subsequently. B. The cell fate of neural stem cells (NSCs) is dependent on the modification status of the Ngn1 promotor and its expression. Methylation on $\mathrm{H} 3$ lysine 4 (H3K4) at the $\mathrm{Ngn1}$ promotor in NSCs leads to expression of the $\mathrm{Ngn} 1$ gene and a neuronal cell fate. However, methylation on H3 lysine 9 (H3K9) leads to repression on $\mathrm{Ngn1}$ and an astrocytic cell fate. C. The knockout of Af9 or Dot1l leads to generation of neurons expressing markers characteristic for lower layers in the cerebral cortex. The number of upper layer neurons and of neural stem cells in the progenitor zone are reduced.

it only requires the removal of the repressive histone methylation for the activation of the gene, as the activating information is already in place. This bivalent state therefore allows a fast temporal activation of gene expression which is of crucial importance for proper differentiation (Harikumar and Meshorer, 2015; Hirabayashi and Gotoh, 2010).

Furthermore, the establishment of one particular histone mark can positively or negatively influence the deposition of a second. An example of this is the ubiquitination of H2B, which facilitates di-methylation of H3K79 by the methyltransferase Disruptor of Teleomeric Silencing-like 1 (DOT1L) (Figure 3A). This concept is known as "histone crosstalk" (Suganuma and Workman, 2008; Nguyen and Zhang, 2011; Zhang et al., 2015.)

Activation but also repression of genes is required for cell differentiation. Both events must occur in a correct temporo-spatial manner to ensure proper differentiation.
Many transcription factors orchestrate gene expression for which they need to access the chromatin and to interact e.g. with enhancer or promoter regions. Thereby, histone modifications are important upstream regulators, since they will regulate the accessibility of the chromatin. Beside histone modifications there are other molecular mechanisms like non coding RNAs, or DNA methylation, by which the activity of transcription factors and chromatin accessibility are regulated additionally (Imamura et al., 2014). 


\section{Histone modifications during neural cell differentiation}

During development and differentiation the fate potential of stem cells becomes sequentially more restricted (Hirabayashi and Gotoh, 2010; Lomvardas and Maniatis, 2016). As mentioned above, neural stem cells differentiate early in development into neurons and in later developmental stages they acquire the potential to differentiate into astrocytes. The switch of the transcriptional program underlying the differentiation into a different cell type is accompanied by chromatin modifications. More specifically, genes involved in differentiation become activated depending on the histone modifications located on the respective promoter. An example of this is, Ngn1 (Neurogenin 1), a gene which is only expressed in neural stem cells that are committed to acquire a neuronal cell fate (neurogenic neural stem cells) (Hirabayashi and Gotoh, 2010; Hirabayashi et al., 2004). At the embryonic stem cell stage, the promoter of Ngn1 contains repressive and activating marks (H3K27me3 and H3K4me3, respectively). The repressive mark H3K9me3 is also present at the Ngn1 promoter at this stage. During differentiation, this bivalent (poised) state of the promoter is resolved by removing the repressive marks. In such case, embryonic stem cells differentiate into neurons where $\mathrm{Ngn1}$ is active (Hirabayashi and Gotoh, 2010; Mikkelsen et al., 2007). In contrast, if Ngn1 stays repressed, the neural stem cells differentiate into astrocytes (Figure 3B). The epigenetic regulation of Ngn1 represents an example of how a poised promoter can resolve into a defined state (active or repressed) with consequences for cell type commitment (neuron or astrocyte).

Another example is the promotor of the Gfap gene that encodes for the astrocytic protein GFAP (Glial fibrillary acidic protein). During astrocyte differentiation this gene is activated, whereas it is repressed in neurons. During astrocyte differentiation, the repressive H3K9me3 at the Gfap promoter is replaced by the activating H3K4me3 (Song and Ghosh, 2004; Takouda et al., 2017).

\section{Histone methylations in central nervous system function and disease}

A growing body of data shows that histone methylations have important impact on central nervous system development and function. E.g. postmortem studies of human brains show that mutations in chromatin writers like
Euchromatic Histone Lysine Methyltransferase 1 (EHMT1), an H3K9 methyltransferase, and Lysine Demethylase 5C (KDM5C), an H3K4 demethylase, are linked to mental retardation (Akbarian and Huang, 2009). The promoter of glutamate receptor subunits (NMDA2), which are critical for excitatory synapses, have the activating marks H3K4me2/me3 and lack the repressive mark H3K27me3 in those regions of the brain and at specific ages of development in which these genes are active. Thus, neuronal activity and development hijack histone methylations to regulate gene expression in a region specific way (Stadler et al., 2005). Further, H3K4me3 levels are decreased in schizophrenia when analysed in human frontal lobe samples (Huang et al., 2007). Spinal cord pathologies such as neural tube defects arise through developmental defects at a time when the neural tube closes (Greene and Copp, 2014). In humans, maternal exposure to compounds that affect epigenetic mechanisms provoke neural tube defects and other behavioural disorders in the offspring (Banik et al., 2017). For example folic acid (Vitamin B9) is routinely administrated to women during pregnancy, to reduce the risk of neural tube defects in the child (Bhargava and Tyagi, 2014; Blom, 2009). Folic acid is used for the conversion of homocysteine to methionine, which can be methlated to S-adenosyl-methionine (SAM). SAM provides the methyl group for methylation reactions, including methylation of histones. Thus, low levels of folic acid can result in a reduction of SAM, and hypomethylation of histones. As we have outlined above, proper histone methylation secures correct activation of transcriptional programs in various cell states. Indeed, there is emerging evidence for a correlation between neural tube defects and aberrant histone methylation such as H3K79me (Wilde et al., 2014; Zhang et al., 2013a). H3K79me levels in neural tube defect patients were significantly lower compared to healthy humans (Zhang et al., 2013b). This result, together with our observations that DOT1L is involved in neuronal differentiation, makes DOT1L and H3K79me important novel targets to study with the aim of designing new and effective therapeutic strategies against neural tube defects. In the following we will therefore enlighten the state-of-the-art on how DOT1L and H3K79me influence central nervous system development.

\section{DOT1L and H3K79 methylation}

In contrast to other methylations described above, H3K79me is not located in the histone tails that stick out of the nucleosome, but it is found in the globular domain 
of $\mathrm{H} 3$ (Figure 2B). H3K79 is mono, di and trimethylated by DOT1L. H3K79me1, me2 or me3 are differently distributed in the coding regions of the genome. H3K79me1 is mainly localised in the gene body, but H3K79me2/3 are more restricted to the transcription starting site (TSS) (Deshpande et al., 2012, 2014). H3K79me2 seems very confined to regions of active transcription (Nguyen and Zhang, 2011; Vlaming and van Leeuwen, 2016). However, not every transcriptional active gene is marked with H3K79me2. In addition, we and others observed that transcriptional activation of specific genes, such as Tbr1 (T Box Brain 1) (Büttner et al., 2010) or ER stress genes in the brain (Roidl et al., 2016), or ENaC $\alpha$ (Scnn1a, Sodium Channel, Nonvoltage-gated 1 alpha) in the kidney (Zhang et al., 2006), comes along with decreased levels of H3K79me2. Thus, the information of H3K79me seems gene specific, dependent on the degree of methylation, and the location within the gene. It is of note that neither eraser nor reader of H3K79me is known as yet, which renders the meaning of this mark still enigmatic.

It has been shown that DOT1L is required for cardiomyocyte differentiation, heart development, hematopoiesis and bone development (Cattaneo et al., 2016; Monteagudo et al., 2017). DOT1L is crucial for stem cell differentiation e.g. into neurons, however it is not required for maintaining stem cell pluripotency (Barry et al., 2009). Recent studies showed that DOT1L is involved in the process of cell aging (through mechanisms not yet understood) and its inhibition facilitates cell reprogramming (Onder et al., 2012; Soria-Valles et al., 2015a, 2015b). Our own work demonstrated that DOT1L and H3K79me promote proliferation of neural stem cells from the cerebral cortex and that DOT1L activity represses endoplasmic reticulum (ER) stress gene transcription in vitro (Roidl et al., 2016). In vivo, DOT1L influences transcriptional programs that are necessary to allow differentiation of specific neuronal subtypes, which reside in different neuronal layers within the cerebral cortex and which are generated at different developmental time points (Büttner et al., 2010, Franz et al., in revision) (Figure 3C). DOT1L is, as mentioned above, required for differentiation of different cell types in various organ systems. But the way in which the H3K79me mark is interpreted by the transcriptional machinery, remains under debate and is still not resolved as yet (Vlaming and van Leeuwen, 2016).

\section{Recruitment of DOT1L to the chromatin through chromatin readers}

DOT1L interacts with other proteins to access specific regions of the genome. For example, DOT1L interacts with RNA polymerase II, which might explain the increased H3K79me near the TSS or H3K79me distribution over entire coding regions (Kim et al., 2012). Mohan et al., 2010 reported that DOT1L forms a protein complex known as DotCom. This complex includes two interactors of DOT1L namely Myeloid/Lymphoid or Mixed-Lineage Leukemia 3 (MLLT3/AF9) and 10 (MLLT10/AF10) which are able to "read" different states of the chromatin. AF9 binds acetylated histones (Li et al., 2014), whereas AF10 binds unmodified H3K27 (Chen et al., 2015). In this way, AF9 and AF10 are recruited to specific genomic regions, and through their interaction with DOT1L the respective chromatin is methylated at H3K79. This crosstalk between acetylated and H3K79me regions is an important mechanism in leukaemia, where fusion proteins of MLL and AF9 or AF10 confer arbitrary presence of DOT1L at genes that drive tumour development and progression (Chen et al., 2013; Deshpande et al., 2012, 2014; Kuntimaddi et al., 2015). We reported that AF9 recruits DOT1L to the chromatin in the cerebral cortex, and that AF9-DOT1L interaction is responsible for repressing TBR1 expression in the developing brain (Büttner et al., 2010). In this way, by repressing TBR1 expression DOT1L may be promoting a neuronal fate commitment towards an upper layer identity (Büttner et al., 2010; Franz et al., in revision).

There are further examples of crosstalks between H3K79me and other histone modifications. Ubiquitination of histone 2B (H2Bub) facilitates methylation of H3K79 by DOT1L (McGinty et al., 2008; Mohan et al., 2010; Wang et al., 2013). This mechanism of DOT1L recruitment is not yet explored in differentiating neurons, even though we showed that H2Bub is required for neural stem cell differentiation. Interestingly RNF40, the ubiquitin ligase involved in $\mathrm{H} 2 \mathrm{Bub}$, is also necessary to resolve a bivalent state (H3K4me3/H3K27me3) of neuronal lineage specific genes towards activation (H3K4me3) (Karpiuk et al., 2012).

\section{Concluding remarks}

Histone methylations play important roles in neural differentiation and central nervous system development. Because embryogenesis is a very complex process it can 
be disturbed in various ways. Therefore, anything that disturbs these epigenetic mechanisms, e.g. mutations in genes coding for chromatin modifiers, readers and erasers, might impact on proper development. This statement is not only valid for development of the central nervous system, since the relevance of chromatin remodelling mechanisms has been shown during the differentiation of other tissues as well (Ghayor and Weber, 2016; Martinez et al., 2015). As a consequence of these discoveries, more techniques and approaches are being developed to specifically study chromatin modifying and remodelling mechanisms (Stricker et al., 2017). Understanding in detail how histone modifications influence development is not only of relevance to understand general principles of gene expression control, but it is also a promising platform for drug discovery, and development of preventive strategies and therapeutic treatments for various diseases including neurological and psychiatric pathologies.

Acknowledgments: We acknowledge the Deutsche Forschungsgemeinschaft funded CRC992 Medical Epigenetics for funding to TV (A03) and IBRO (International Brain Research Organization), DAAD (Deutscher Akademisher Austausch Dienst) and the Argentinean Ministry of Education for the postdoctoral scholarships awarded to AV.

\section{Bibliography}

Akbarian, S., and Huang, H.-S. (2009). Epigenetic Regulation in Human Brain - Focus on Histone Lysine Methylation. Biol. Psychiatry 65, 198-203.

Banik, A., Kandilya, D., Ramya, S., Stünkel, W., Chong, Y. S., and Dheen, S. T. (2017). Maternal Factors that Induce Epigenetic Changes Contribute to Neurological Disorders in Offspring. Genes 8.

Barry, E. R., Krueger, W., Jakuba, C. M., Veilleux, E., Ambrosi, D. J., Nelson, C. E., and Rasmussen, T. P. (2009). ES cell cycle progression and differentiation require the action of the histone methyltransferase Dot1L. Stem Cells Dayt. Ohio 27, 1538-1547.

Bhargava, S., and Tyagi, S. C. (2014). Nutriepigenetic regulation by folate-homocysteine-methionine axis: a review. Mol. Cell. Biochem. 387, 55-61.

Blom, H. J. (2009). Folic acid, methylation and neural tube closure in humans. Birt. Defects Res. A. Clin. Mol. Teratol. 85, 295-302.

Büttner, N., Johnsen, S. A., Kügler, S., and Vogel, T. (2010). Af9/Mllt3 interferes with Tbr1 expression through epigenetic modification of histone H3K79 during development of the cerebral cortex. Proc. Natl. Acad. Sci. U.S.A. 107, 7042-7047.

Cattaneo, P., Kunderfranco, P., Greco, C., Guffanti, A., Stirparo, G. G., Rusconi, F., Rizzi, R., Di Pasquale, E., Locatelli, S. L., Latronico, M. V. G., et al. (2016). DOT1L-mediated H3K79me2 modification critically regulates gene expression during cardiomyocyte differentiation. Cell Death Differ. 23, 555-564.

Chen, L., Deshpande, A., Banka, D., Bernt, K. M., Dias, S., Buske, C., Olhava, E. J., Daigle, S. R., Richon, V. M., Pollock, R. M., et al. (2013). Abrogation of MLL-AF10 and CALM-AF10 mediated transformation through genetic inactivation or pharmacological inhibition of the H3K79 methyltransferase Dot1l. Leukemia 27, 813-822.

Chen, S., Yang, Z., Wilkinson, A. W., Deshpande, A. J., Sidoli, S., Krajewski, K., Strahl, B. D., Garcia, B. A., Armstrong, S. A., Patel, D. J., et al. (2015). The PZP Domain of AF10 Senses Unmodified H3K27 to Regulate DOT1L-Mediated Methylation of H3K79. Mol. Cell 60, 319-327.

Cutter, A. R., and Hayes, J. J. (2015). A brief review of nucleosome structure. FEBS Lett. 589, 2914-2922.

Deshpande, A. J., Bradner, J., and Armstrong, S. A. (2012). Chromatin modifications as therapeutic targets in MLL-rearranged Leukemia. Trends Immunol. 33, 563-570.

Deshpande, A. J., Deshpande, A., Sinha, A. U., Chen, L., Chang, J., Cihan, A., Fazio, M., Chen, C.-W., Zhu, N., Koche, R., et al. (2014). AF10 regulates progressive H3K79 methylation and HOX gene expression in diverse AML subtypes. Cancer Cell 26, 896-908.

Ghayor, C., and Weber, F. E. (2016). Epigenetic Regulation of Bone Remodeling and Its Impacts in Osteoporosis. Int. J. Mol. Sci. 17. Greene, N. D. E., and Copp, A. J. (2014). Neural tube defects. Annu. Rev. Neurosci. 37, 221-242.

Harikumar, A., and Meshorer, E. (2015). Chromatin remodeling and bivalent histone modifications in embryonic stem cells. EMBO Rep. 16, 1609-1619.

Heintzman, N. D., Stuart, R. K., Hon, G., Fu, Y., Ching, C. W., Hawkins, R. D., Barrera, L. O., Van Calcar, S., Qu, C., Ching, K. A., et al. (2007). Distinct and predictive chromatin signatures of transcriptional promoters and enhancers in the human genome. Nat. Genet. 39, 311-318.

Hirabayashi, Y., and Gotoh, Y. (2010). Epigenetic control of neural precursor cell fate during development. Nat. Rev. Neurosci. 11, 377-388.

Hirabayashi, Y., Itoh, Y., Tabata, H., Nakajima, K., Akiyama, T., Masuyama, N., and Gotoh, Y. (2004). The Wnt/beta-catenin pathway directs neuronal differentiation of cortical neural precursor cells. Dev. Camb. Engl. 131, 2791-2801.

Huang, H.-S., Matevossian, A., Whittle, C., Kim, S. Y., Schumacher, A., Baker, S. P., and Akbarian, S. (2007). Prefrontal Dysfunction in Schizophrenia Involves Mixed-Lineage Leukemia 1-Regulated Histone Methylation at GABAergic Gene Promoters. J. Neurosci. 27, 11254-11262.

Imamura, T., Uesaka, M., and Nakashima, K. (2014). Epigenetic setting and reprogramming for neural cell fate determination and differentiation. Philos. Trans. R. Soc. B Biol. Sci. 369.

Karpiuk, O., Najafova, Z., Kramer, F., Hennion, M., Galonska, C., König, A., Snaidero, N., Vogel, T., Shchebet, A., Begus-Nahrmann, Y., et al. (2012). The Histone H2B Monoubiquitination Regulatory Pathway Is Required for Differentiation of Multipotent Stem Cells. Mol. Cell 46, 705-713.

Kiecker, C., Bates, T., and Bell, E. (2016). Molecular specification of germ layers in vertebrate embryos. Cell. Mol. Life Sci. CMLS 73, 923-947.

Kim, S.-K., Jung, I., Lee, H., Kang, K., Kim, M., Jeong, K., Kwon, C. S., Han, Y.-M., Kim, Y. S., Kim, D., et al. (2012). Human 
histone H3K79 methyltransferase DOT1L protein [corrected] binds actively transcribing RNA polymerase II to regulate gene expression. J. Biol. Chem. 287, 39698-39709.

Kuntimaddi, A., Achille, N. J., Thorpe, J., Lokken, A. A., Singh, R., Hemenway, C. S., Adli, M., Zeleznik-Le, N. J., and Bushweller, J. H. (2015). Degree of Recruitment of DOT1L to MLL-AF9 Defines Level of H3K79 Di- and Tri-methylation on Target Genes and Transformation Potential. Cell Rep. 11, 808-820.

Lawrence, M., Daujat, S., and Schneider, R. (2016). Lateral Thinking: How Histone Modifications Regulate Gene Expression. Trends Genet. TIG 32, 42-56.

Li, Y., Wen, H., Xi, Y., Tanaka, K., Wang, H., Peng, D., Ren, Y., Jin, Q., Dent, S. Y. R., Li, W., et al. (2014). AF9 YEATS Domain Links Histone Acetylation to DOT1L-Mediated H3K79 Methylation. Cell 159, 558-571.

Lomvardas, S., and Maniatis, T. (2016). Histone and DNA Modifications as Regulators of Neuronal Development and Function. Cold Spring Harb. Perspect. Biol. 8.

Luger, K., Dechassa, M. L., and Tremethick, D. J. (2012). New insights into nucleosome and chromatin structure: an ordered state or a disordered affair? Nat. Rev. Mol. Cell Biol. 13, 436-447.

Martinez, S. R., Gay, M. S., and Zhang, L. (2015). Epigenetic mechanisms in heart development and disease. Drug Discov. Today 20, 799-811.

McGinty, R. K., Kim, J., Chatterjee, C., Roeder, R. G., and Muir, T. W. (2008). Chemically ubiquitylated histone H2B stimulates hDot1L-mediated intranucleosomal methylation. Nature 453 , 812-816.

Mikkelsen, T. S., Ku, M., Jaffe, D. B., Issac, B., Lieberman, E., Giannoukos, G., Alvarez, P., Brockman, W., Kim, T.-K., Koche, R. P., et al. (2007). Genome-wide maps of chromatin state in pluripotent and lineage-committed cells. Nature 448, 553.

Mohan, M., Herz, H.-M., Takahashi, Y.-H., Lin, C., Lai, K. C., Zhang, Y., Washburn, M. P., Florens, L., and Shilatifard, A. (2010). Linking H3K79 trimethylation to Wnt signaling through a novel Dot1-containing complex (DotCom). Genes Dev. 24, 574-589.

Monteagudo, S., Cornelis, F. M. F., Aznar-Lopez, C., Yibmantasiri, P., Guns, L.-A., Carmeliet, P., Cailotto, F., and Lories, R. J. (2017). DOT1L safeguards cartilage homeostasis and protects against osteoarthritis. Nat. Commun. 8, 15889.

Nguyen, A. T., and Zhang, Y. (2011). The diverse functions of Dot1 and H3K79 methylation. Genes Dev. 25, 1345-1358.

Onder, T. T., Kara, N., Cherry, A., Sinha, A. U., Zhu, N., Bernt, K. M., Cahan, P., Marcarci, B. O., Unternaehrer, J., Gupta, P. B., et al. (2012). Chromatin-modifying enzymes as modulators of reprogramming. Nature 483, 598-602.

Peserico, A., and Simone, C. (2011). Physical and Functional HAT/ HDAC Interplay Regulates Protein Acetylation Balance. J. Biomed. Biotechnol. 2011.

Peterson, C. L., and Laniel, M.-A. (2004). Histones and histone modifications. Curr. Biol. 14, R546-R551.

Prachayasittikul, V., Prathipati, P., Pratiwi, R., Phanus-Umporn, C., Malik, A. A., Schaduangrat, N., Seenprachawong, K., Wongchitrat, P., Supokawej, A., Prachayasittikul, V., et al. (2017). Exploring the epigenetic drug discovery landscape. Expert Opin. Drug Discov. 12, 345-362.

Qiao, Y., Yang, X., and Jing, N. (2016). Epigenetic regulation of early neural fate commitment. Cell. Mol. Life Sci. 73, 1399-1411.

Roidl, D., Hellbach, N., Bovio, P. P., Villarreal, A., Heidrich, S., Nestel, S., Grüning, B. A., Boenisch, U., and Vogel, T. (2016). DOT1L
Activity Promotes Proliferation and Protects Cortical Neural Stem Cells from Activation of ATF4-DDIT3-Mediated ER Stress In Vitro. Stem Cells Dayt. Ohio 34, 233-245.

Shlyueva, D., Stampfel, G., and Stark, A. (2014). Transcriptional enhancers: from properties to genome-wide predictions. Nat. Rev. Genet. 15, 272-286.

Smith, J. L., and Schoenwolf, G. C. (1997). Neurulation: coming to closure. Trends Neurosci. 20, 510-517.

Solnica-Krezel, L., and Sepich, D. S. (2012). Gastrulation: making and shaping germ layers. Annu. Rev. Cell Dev. Biol. 28, 687-717.

Song, M.-R., and Ghosh, A. (2004). FGF2-induced chromatin remodeling regulates CNTF-mediated gene expression and astrocyte differentiation. Nat. Neurosci. 7, 229-235.

Soria-Valles, C., Osorio, F. G., Gutiérrez-Fernández, A., De Los Angeles, A., Bueno, C., Menéndez, P., Martín-Subero, J. I., Daley, G. Q., Freije, J. M. P., and López-Otín, C. (2015a). NF-кB activation impairs somatic cell reprogramming in ageing. Nat. Cell Biol. 17, 1004-1013.

Soria-Valles, C., Osorio, F. G., and López-Otín, C. (2015b). Reprogramming aging through DOT1L inhibition. Cell Cycle 14, 3345-3346.

Stadler, F., Kolb, G., Rubusch, L., Baker, S. P., Jones, E. G., and Akbarian, S. (2005). Histone methylation at gene promoters is associated with developmental regulation and region-specific expression of ionotropic and metabotropic glutamate receptors in human brain. J. Neurochem. 94, 324-336.

Stricker, S. H., Köferle, A., and Beck, S. (2017). From profiles to function in epigenomics. Nat. Rev. Genet. 18, 51-66.

Suganuma, T., and Workman, J. L. (2008). Crosstalk among Histone Modifications. Cell 135, 604-607.

Takouda, J., Katada, S., and Nakashima, K. (2017). Emerging mechanisms underlying astrogenesis in the developing mammalian brain. Proc. Jpn. Acad. Ser. B Phys. Biol. Sci. 93, 386-398.

Tang, K., Peng, G., Qiao, Y., Song, L., and Jing, N. (2015). Intrinsic regulations in neural fate commitment. Dev. Growth Differ. 57, 109-120.

Tseng, W.-C., Munisha, M., Gutierrez, J. B., and Dougan, S. T. (2017) Establishment of the Vertebrate Germ Layers. Adv. Exp. Med. Biol. 953, 307-381.

Venkatesh, S., and Workman, J. L. (2015). Histone exchange, chromatin structure and the regulation of transcription. Nat. Rev. Mol. Cell Biol. 16, 178-189.

Vlaming, H., and van Leeuwen, F. (2016). The upstreams and downstreams of H3K79 methylation by DOT1L. Chromosoma.

Wang, E., Kawaoka, S., Yu, M., Shi, J., Ni, T., Yang, W., Zhu, J., Roeder, R. G., and Vakoc, C. R. (2013). Histone H2B ubiquitin ligase RNF20 is required for MLL-rearranged leukemia. Proc. Natl. Acad. Sci. U.S.A. 110, 3901-3906.

Wilde, J. J., Petersen, J. R., and Niswander, L. (2014). Genetic, epigenetic, and environmental contributions to neural tube closure. Annu. Rev. Genet. 48, 583-611.

Yang, X.-J., and Seto, E. (2007). HATs and HDACs: from structure, function and regulation to novel strategies for therapy and prevention. Oncogene 26, 5310-5318.

Zhang, Q., Xue, P., Li, H., Bao, Y., Wu, L., Chang, S., Niu, B., Yang, F., and Zhang, T. (2013a). Histone modification mapping in human brain reveals aberrant expression of histone $\mathrm{H} 3$ lysine 
79 dimethylation in neural tube defects. Neurobiol. Dis. 54, 404-413.

Zhang, Q., Xue, P., Li, H., Bao, Y., Wu, L., Chang, S., Niu, B., Yang, F., and Zhang, T. (2013b). Histone modification mapping in human brain reveals aberrant expression of histone $\mathrm{H} 3$ lysine 79 dimethylation in neural tube defects. Neurobiol. Dis. 54, 404-413.

Zhang, T., Cooper, S., and Brockdorff, N. (2015). The interplay of histone modifications - writers that read. EMBO Rep.16, $1467-1481$.

Zhang, W., Xia, X., Jalal, D. I., Kuncewicz, T., Xu, W., Lesage, G. D., and Kone, B. C. (2006). Aldosterone-sensitive repression of ENaCalpha transcription by a histone $\mathrm{H} 3$ lysine-79 methyltransferase. Am. J. Physiol. Cell Physiol. 290, C936-946.

Article note: German version available at https://doi.org/10.1515/nf2018-0001

\section{Bionotes}

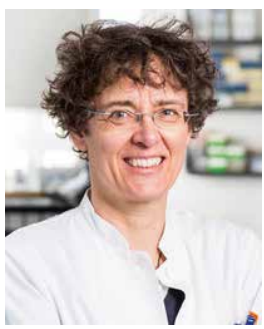

\section{Prof. Dr. Tanja Vogel}

Institute for Anatomy and Cell Biology, Department of Molecular Embryology, Medical Faculty, Albert-Ludwigs-University Freiburg, 79104 Freiburg, Germany, Phone: +49 761 203-5086 Mail: Tanja.Vogel@anat.uni-freiburg.de

Prof. Dr. Tanja Vogel studied biochemistry at the University of Hanover (1994). rer. nat. at the Institute of Human Genetics at the Hannover Medical School (1997). She spent her time as a postdoc at the MRC Human Genetics Unit in Edinburgh and then the MPI for Biophysical Chemistry in Göttingen. From 2005 she was group leader in the Department of Neuroanatomy at the Center for Anatomy of the University Medicine of Göttingen. She completed her habilitation in 2010 in the Department of Anatomy. For her habilitation she was awarded the distinction of the best habilitation of the Medical Faculty. For the summer semester 2011, Prof. Vogel moved to the University of Freiburg. Since 2014 she has been Acting Director of the Department of Molecular Embryology at the Institute of Anatomy and Cell Biology. Prof. Vogel's research interests lie in the field of neurobiology: epigenetic histone modifications in CNS development and function, TGFß signals of neuronal stem cell differentiation and non-coding RNAs in neurological developmental disorders.

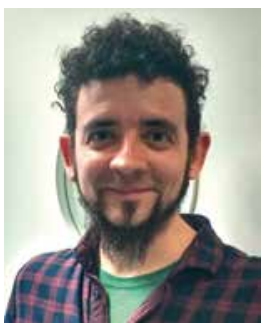

\section{Alejandro Villarreal}

Institute for Anatomy and Cell Biology, Department of Molecular Embryology, Medical Faculty, Albert-Ludwigs-University Freiburg, 79104 Freiburg, Germany, Phone: +49 761 203-5108

Mail: Alejandro.Villarreal@anat.uni-freiburg.de

Mr. Dr. Alejandro Villarreal studied biology at Buenos Aires University. He received his doctorate in 2014 with Dr. Alberto Javier Ramos with a study on reactive astrocytes in a brain stroke model. He joined Prof. Dr Vogel's group as a postdoctoral researcher to gain expertise in epigenetics and histone modification research. His research interests include neural cell differentiation, developmental biology and brain injury.

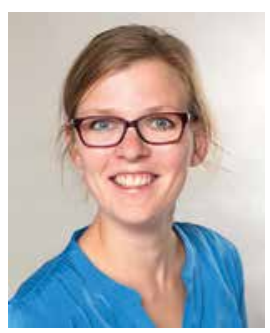

\section{Dr. Henriette Franz} Institute for Anatomy and Cell Biology, Department of Molecular Embryology, Medical Faculty, Albert-Ludwigs-University Freiburg, 79104 Freiburg, Germany, Phone: +49 761 203-5108

Mail: Henriette.Franz@anat.uni-freiburg.de

Dr. Henriette Franz graduated in Biochemsitry at the University of Leipzig in 2005. She earned her PhD at the Max Planck Institute for Biophysical Chemistry, Göttingen in 2010. For her postdoctoral specialization, Dr. Franz moved to the Center for Clinical Research at the University of Freiburg. Since 2015 she is conducting research and teaching in the field of Neuroepigenetics at the Department of Molecular Embryology at the Institute of Anatomy and Cell Biology in Freiburg. 
